\title{
Erratum: Naturalistic Affective Expression Classification by a Multi-stage Approach Based on Hidden Markov Models
}

\author{
Hongying Meng and Nadia Bianchi-Berthouze \\ UCL Interaction Centre, University College London, London, UK \\ h.meng@ucl.ac.uk, n.berthouze@ucl.ac.uk
}

S. D’Mello et al. (Eds.): ACII 2011, Part II, LNCS 6975, pp. 378-387, 2011.

(C) Springer-Verlag Berlin Heidelberg 2011

DOI: 10.1007/978-3-642-24571-8_75

The acknowledgement text of the initially published paper was incomplete. It should have been as follows:

This work was supported by EPSRC grant EP/G043507/1: Pain rehabilitation: E/Motion-based automated coaching. This work was funded by the EPSRC Emotion \& Pain Project EP/H017178/1. 